\title{
LENGTHS, AREA AND MODULUS OF CONTINUITY OF SOME CLASSES OF COMPLEX-VALUED FUNCTIONS
}

\author{
SHAOLIN CHEN
}

\begin{abstract}
In this paper, we discuss the modulus of continuity of solutions to Poisson's equation, and give bounds of length and area distortion for some classes of $K$-quasiconformal mappings satisfying Poisson's equations. The obtained results are the extension of the corresponding classical results.
\end{abstract}

\section{Preliminaries AND main Results}

We use $\mathbb{C}$ to denote the complex plane. For $a \in \mathbb{C}$ and $r>0$, let $\mathbb{D}(a, r)=\{z$ : $|z-a|<r\}, \mathbb{D}_{r}=\mathbb{D}(0, r)$ and $\mathbb{D}=\mathbb{D}_{1}$, the open unit disk in $\mathbb{C}$. Let $\mathbb{T}=\partial \mathbb{D}$ be the boundary of $\mathbb{D}$. Furthermore, we denote by $\mathcal{C}^{m}(\Omega)$ the set of all complex-valued $m$ times continuously differentiable functions from $\Omega$ into $\mathbb{C}$, where $\Omega$ is a subset of $\mathbb{C}$ and $m \in\{0,1,2, \ldots\}$. In particular, $\mathcal{C}(\Omega):=\mathcal{C}^{0}(\Omega)$ denotes the set of all continuous functions in $\Omega$. Let $G$ be a domain of $\mathbb{C}$, and let $\bar{G}$ be the closure of $G$. We use $d_{G}(z)$ to denote the Euclidean distance from $z$ to the boundary $\partial G$ of $G$. Especially, we always use $d(z)$ to denote the Euclidean distance from $z$ to the boundary of $\mathbb{D}$.

For a real $2 \times 2$ matrix $A$, we use the matrix norm

$$
\|A\|=\sup \{|A z|:|z|=1\}
$$

and the matrix function

$$
\lambda(A)=\inf \{|A z|:|z|=1\} .
$$

For $z=x+i y \in \mathbb{C}$, the formal derivative of a complex-valued function $f=u+i v$ is given by

$$
D_{f}=\left(\begin{array}{ll}
u_{x} & u_{y} \\
v_{x} & v_{y}
\end{array}\right) \text {, }
$$

so that

$$
\left\|D_{f}\right\|=\left|f_{z}\right|+\left|f_{\bar{z}}\right| \text { and } \lambda\left(D_{f}\right)=|| f_{z}|-| f_{\bar{z}}||
$$

where

$$
f_{z}=\frac{1}{2}\left(f_{x}-i f_{y}\right) \text { and } f_{\bar{z}}=\frac{1}{2}\left(f_{x}+i f_{y}\right) .
$$

We use

$$
J_{f}:=\operatorname{det} D_{f}=\left|f_{z}\right|^{2}-\left|f_{\bar{z}}\right|^{2}
$$

to denote the Jacobian of $f$.

File: C-final-2019-2-11.tex, printed: 19-3-2019, 0.53

2000 Mathematics Subject Classification. Primary: 31A05; Secondary: 30H30.

Key words and phrases. Length, Area, Modulus of continuity, Poisson's equation. 
For $z, w \in \mathbb{D}$, let

$$
G(z, w)=\log \left|\frac{1-z \bar{w}}{z-w}\right|
$$

and

$$
P\left(z, e^{i \theta}\right)=\frac{1-|z|^{2}}{\left|1-z e^{-i \theta}\right|^{2}}
$$

denote the Green function and (harmonic) Poisson kernel, respectively, where $\theta \in$ $[0,2 \pi]$.

Let $\psi: \mathbb{T} \rightarrow \mathbb{C}$ be a bounded integrable function and let $g \in \mathcal{C}(\overline{\mathbb{D}})$. For $z \in \mathbb{D}$, the solution to the Poisson's equation

$$
\Delta f(z)=g(z)
$$

satisfying the boundary condition $\left.f\right|_{\mathbb{T}}=\psi \in L^{1}(\mathbb{T})$ is given by

$$
f(z)=P[\psi](z)-G[g](z)
$$

where

$$
G[g](z)=\frac{1}{2 \pi} \int_{\mathbb{D}} G(z, w) g(w) d A(w), P[\psi](z)=\frac{1}{2 \pi} \int_{0}^{2 \pi} P\left(z, e^{i t}\right) \psi\left(e^{i t}\right) d t,
$$

and $d A(w)$ denotes the Lebesgue measure on $\mathbb{D}$. It is well known that if $\psi$ and $g$ are continuous in $\mathbb{T}$ and in $\overline{\mathbb{D}}$, respectively, then $f=P[\psi]-G[g]$ has a continuous extension $\tilde{f}$ to the boundary, and $\tilde{f}=\psi$ in $\mathbb{T}$ (see [12, pp. 118-120] and [13, 14]).

A continuous increasing function $\omega:[0, \infty) \rightarrow[0, \infty)$ with $\omega(0)=0$ is called a majorant if $\omega(t) / t$ is non-increasing for $t>0$. Given a subset $\Omega$ of $\mathbb{C}$, a function $f: \Omega \rightarrow \mathbb{C}$ is said to belong to the Lipschitz space $\mathcal{L}_{\omega}(\Omega)$ if there is a positive constant $C$ such that

$$
|f(z)-f(w)| \leq C \omega(|z-w|) \text { for all } z, w \in \Omega .
$$

For $\delta_{0}>0$, let

$$
\int_{0}^{\delta} \frac{\omega(t)}{t} d t \leq C \cdot \omega(\delta), 0<\delta<\delta_{0}
$$

and

$$
\delta \int_{\delta}^{+\infty} \frac{\omega(t)}{t^{2}} d t \leq C \cdot \omega(\delta), 0<\delta<\delta_{0},
$$

where $\omega$ is a majorant and $C$ is a positive constant.

A majorant $\omega$ is said to be regular if it satisfies the conditions (1.6) and (1.7) (see $[8,9,20])$.

Let $G$ be a proper subdomain of $\mathbb{C}$. We say that a function $f$ belongs to the local Lipschitz space $\operatorname{loc} \mathcal{L}_{\omega}(G)$ if (1.5) holds, with a fixed positive constant $C$, whenever $z \in G$ and $|z-w|<\frac{1}{2} d_{G}(z)$ (cf. [10, 16]). Moreover, $G$ is said to be a 
$\mathcal{L}_{\omega}$-extension domain if $\mathcal{L}_{\omega}(G)=\operatorname{loc} \mathcal{L}_{\omega}(G)$. The geometric characterization of $\mathcal{L}_{\omega^{-}}$ extension domains was given by Gehring and Martio [10]. Then Lappalainen [16] generalized their characterization, and proved that $G$ is a $\mathcal{L}_{\omega}$-extension domain if and only if each pair of points $z, w \in G$ can be joined by a rectifiable curve $\gamma \subset G$ satisfying

$$
\int_{\gamma} \frac{\omega\left(d_{G}(\zeta)\right)}{d_{G}(\zeta)} d s(\zeta) \leq C \omega(|z-w|)
$$

with some fixed positive constant $C=C(G, \omega)$, where $d s$ is the arc length measure on $\gamma$. Furthermore, Lappalainen [16, Theorem 4.12] showed that $\mathcal{L}_{\omega}$-extension domains exist only for majorants $\omega$ satisfying (1.6).

The following result is the classical Hardy-Littlewood type Theorem for analytic functions with respect to the majorant $\omega(t)=\omega_{\alpha}(t)=t^{\alpha}(0<\alpha \leq 1)$ for $t \in$ $[0,+\infty)$. In fact, the Hardy-Littlewood type Theorems and the modulus of continuity of analytic functions are closely related.

Theorem A. ([7, Theorem 5.1]) Let $f$ be an analytic function in $\mathbb{D}$ and continuous in $\overline{\mathbb{D}}$. Then

$$
\left|f\left(e^{i \theta_{1}}\right)-f\left(e^{i \theta_{2}}\right)\right| \leq C \omega_{\alpha}\left(\left|\theta_{1}-\theta_{2}\right|\right) \text { for all } 0 \leq \theta_{1}, \theta_{2}<2 \pi
$$

if and only if

$$
\left|f^{\prime}(z)\right| \leq C \frac{\omega_{\alpha}(d(z))}{d(z)} \text { for all } z \in \mathbb{D}
$$

where $C$ is a positive constant.

Krantz [15] established the following Hardy-Littlewood type theorem for real harmonic functions.

Theorem B. ([15, Theorem 15.8]) Let $u$ be a real harmonic function in $\mathbb{D}$, and $\omega(t)=\omega_{\alpha}(t)=t^{\alpha}$ be a majorant for $0<\alpha \leq 1$. Then $u$ satisfies

$$
|\nabla u(z)| \leq C \frac{\omega_{\alpha}(d(z))}{d(z)} \text { for all } z \in \mathbb{D}
$$

if and only if

$$
|u(z)-u(w)| \leq C \omega_{\alpha}(|z-w|) \text { for all } z, w \in \mathbb{D},
$$

where $C$ is a positive constant.

Moduli of continuity of harmonic quasiregular mappings via Hardy-Littlewood property is considered in [1]. In [17], the authors characterizes the moduli of continuity of a function $f$ by using the square of distance function and module of $\Delta f$ (see the the class $O C^{2}(G)$ in [17]). In particular, quasiregular versions of the well-known result due to Koebe, [18, Theorem 4.2], is established and, by using this result, an extension of Dyakonov's theorem for quasiregular mappings in space (without Dyakonov's hypothesis that it is a quasiregular local homeomorphism), [18, Theorem 4.3], is proved. The charcterization of Lipschitz-type spaces for quasiregular mappings by average Jacobian is also established in [18, Theorem 4.3]. 
For a given $g \in \mathcal{C}(\bar{\Omega})$, let

$$
\mathcal{F}_{g}(\Omega)=\left\{f \in \mathcal{C}(\bar{\Omega}) \cap \mathcal{C}^{2}(\Omega): \Delta f(z)=g(z), z \in \Omega\right\},
$$

where $\Omega$ is a proper subdomain of $\mathbb{C}$. Obviously, all analytic functions and harmonic mappings defined in $\bar{\Omega}$ belong to $\mathcal{F}_{0}(\Omega)$. We improve Theorems $\mathrm{A}$ and $\mathrm{B}$ into the following form.

Theorem 1.1. Suppose that $\omega$ is a majorant satisfying (1.6), and $\Omega$ is a bounded $\mathcal{L}_{\omega}$-extension domain. For a given $g \in \mathcal{C}(\bar{\Omega})$, let $f \in \mathcal{F}_{g}(\Omega)$. Then $f \in \mathcal{L}_{\omega}(\Omega)$ if and only if there exists a constant $C>0$ such that, for all $z \in \Omega$,

$$
\left\|D_{f}(z)\right\| \leq C \frac{\omega\left(d_{\Omega}(z)\right)}{d_{\Omega}(z)} .
$$

A mapping $f \in \mathcal{C}^{1}(\mathbb{D})$ is called a Bloch type mapping if $f$ satisfies

$$
\sup _{z \in \mathbb{D}}\left\{\left\|D_{f}(z)\right\| \omega\left((d(z))^{\alpha}\right)\right\}<+\infty,
$$

where $\omega$ is a majorant and $\alpha>0$ is a constant. The set of all Bloch type mappings, denoted by the symbol $\mathcal{B}_{\omega}^{\alpha}$, forms a complex Banach space with the norm $\|\cdot\|$ given by

$$
\|f\|_{\mathcal{B}_{\omega}^{\alpha}}=|f(0)|+\sup _{z \in \mathbb{D}}\left\{\left\|D_{f}(z)\right\| \omega\left((d(z))^{\alpha}\right)\right\} .
$$

In the following, by using the weighted Lipschitz function, Holland and Walsh [11] gave an equivalent characterization of the analytic Bloch space. For the related investigation of this topic for real functions, we refer to $[19,21]$.

Theorem C. ([11, Theorem 3]) Let $f$ be analytic in $\mathbb{D}$, and let $\omega$ be a majorant satisfying $\omega(t)=t$ for $t \in[0,+\infty)$. Then $f \in \mathcal{B}_{\omega}^{1}$ if and only if

$$
\sup _{z, w \in \mathbb{D}, z \neq w}\left\{\frac{\sqrt{\left(1-|z|^{2}\right)\left(1-|w|^{2}\right)}|f(z)-f(w)|}{|z-w|}\right\}<\infty .
$$

In [9], Dyakonov studied the relationship between the modulus of continuity and the bounded mean oscillation on analytic functions in $\mathbb{D}$, and obtained the following result.

Theorem D. ([9, Theorem 1]) Suppose that $f$ is an analytic function in $\mathbb{D}$ which is continuous up to the boundary of $\mathbb{D}$. If $\omega$ and $\omega^{2}$ are regular majorants, then

$$
f \in \mathcal{L}_{\omega}(\mathbb{D}) \Longleftrightarrow P\left[|f|^{2}\right](z)-|f(z)|^{2} \leq M \omega^{2}(d(z)) .
$$

Analogy Theorems $\mathrm{C}$ and $\mathrm{D}$, we prove the following result.

Theorem 1.2. For a given $g \in \mathcal{C}(\overline{\mathbb{D}})$, let $f \in \mathcal{F}_{g}(\mathbb{D})$. Then, for $1 \leq \alpha<2$ and $a$ majorant $\omega$, the following statements are equivalent:

(1) $f \in \mathcal{B}_{\omega}^{\alpha}$; 
(2) There exists a constant $C>0$ such that for all $r \in(0, d(z)]$,

$$
\frac{1}{|\mathbb{D}(z, r)|} \int_{\mathbb{D}(z, r)}|f(\zeta)-f(z)| d A(\zeta) \leq C \frac{r}{\omega\left(r^{\alpha}\right)},
$$

where $|\mathbb{D}(z, r)|$ denotes the area of $\mathbb{D}(z, r)$.

By [5, Theorem 3] and Theorem 1.2, we obtain the following result which is a generalization of Theorem $\mathrm{C}$.

Corollary 1.3. For a given $g \in \mathcal{C}(\overline{\mathbb{D}})$, let $f \in \mathcal{F}_{g}(\mathbb{D})$. Then, for $0 \leq s<1$ and $1 \leq \alpha \leq s+1$, the following are equivalent:

(1) $f \in \mathcal{B}_{\omega}^{\alpha}$;

(2) There exists a constant $C>0$ such that for all $r \in(0, d(z)]$,

$$
\frac{1}{|\mathbb{D}(z, r)|} \int_{\mathbb{D}(z, r)}|f(\zeta)-f(z)| d A(\zeta) \leq \frac{C r}{\omega\left(r^{\alpha}\right)}
$$

where $|\mathbb{D}(z, r)|$ denotes the area of $\mathbb{D}(z, r)$;

(3) There exists a constant $C>0$ such that for all $z, w \in \mathbb{D}$ with $z \neq w$,

$$
\frac{|f(z)-f(w)|}{|z-w|} \leq \frac{C}{\omega\left(d^{s}(z) d^{\alpha-s}(w)\right)} .
$$

For $r \in[0,1)$, the perimeter of the curve $C(r)=\left\{w=f\left(r e^{i \theta}\right): \theta \in[0,2 \pi]\right\}$, counting multiplicity, is defined by

$$
\ell_{f}(r)=\int_{0}^{2 \pi}\left|d f\left(r e^{i \theta}\right)\right|=r \int_{0}^{2 \pi}\left|f_{z}\left(r e^{i \theta}\right)-e^{-2 i \theta} f_{\bar{z}}\left(r e^{i \theta}\right)\right| d \theta,
$$

where $f \in \mathcal{C}^{1}(\mathbb{D})$. In particular, let $\ell_{f}(1)=\sup _{0<r<1} \ell_{f}(r)$ (cf. [4]).

A sense-preserving homeomorphic $f$ from a domain $\Omega$ onto $\Omega^{\prime}$, contained in the Sobolev class $W_{\text {loc }}^{1,2}(\Omega)$, is said to be a $K$-quasiconformal mapping if, for $z \in \Omega$,

$$
\left\|D_{f}(z)\right\|^{2} \leq K\left|\operatorname{det} D_{f}(z)\right| \text {, i.e., }\left\|D_{f}(z)\right\| \leq K \lambda\left(D_{f}(z)\right),
$$

where $K \geq 1$ (cf. [13, 14]). In the following, we will give bounds of length and area distortion for some classes of $K$-quasiconformal mappings satisfying Poisson's equations.

Theorem 1.4. For a given $g \in \mathcal{C}(\overline{\mathbb{D}})$, let $f \in \mathcal{F}_{g}(\mathbb{D})$. If $f=P[f]-G[g]$ is a $K$-quasiconformal mapping with $\ell_{f}(1)<+\infty$, then, for $n \geq 1$,

$$
\begin{gathered}
\left|a_{n}\right|+\left|b_{n}\right| \leq \frac{K \ell_{f}(1)}{2 n \pi}+\frac{2}{3 n}\|g\|_{\infty}, \\
\sup _{z \in \mathbb{D}}\left\{\left\|D_{P[f]}(z)\right\|\left(1-|z|^{2}\right)\right\} \leq\left(\frac{\ell_{f}^{2}(1) K}{4 \pi^{2}}+\frac{4}{9}\|g\|_{\infty}^{2}+\frac{\ell_{f}(1) K^{\frac{1}{2}}}{3 \pi}\|g\|_{\infty}\right)^{\frac{1}{2}}
\end{gathered}
$$

and $f \in \mathcal{B}_{\omega}^{1}$, where $P[f](z)=\sum_{n=0}^{\infty} a_{n} z^{n}+\sum_{n=1}^{\infty} \bar{b}_{n} \bar{z}^{n}$ and $\omega(t)=t$. 
In particular, if $K=1$ and $\|g\|_{\infty}=0$, then the estimates (1.10) and (1.11) are sharp, and the extreme function is $f(z)=z$ for $z \in \overline{\mathbb{D}}$.

For $\theta \in[0,2 \pi]$, the radial length of the curve $C_{\theta}(r)=\left\{w=f\left(\rho e^{i \theta}\right): 0 \leq \rho \leq r<\right.$ $1\}$, counting multiplicity, is defined by

$$
\ell_{f}^{*}(r, \theta)=\int_{0}^{r}\left|d f\left(\rho e^{i \theta}\right)\right|=\int_{0}^{r}\left|f_{z}\left(\rho e^{i \theta}\right)+e^{-2 i \theta} f_{\bar{z}}\left(\rho e^{i \theta}\right)\right| d \rho,
$$

where $f \in \mathcal{C}^{1}(\mathbb{D})$ (cf. [6]). In particular, let

$$
\ell_{f}^{*}(1, \theta)=\sup _{0 \leq r<1} \ell_{f}^{*}(r, \theta) .
$$

Theorem 1.5. For a given $g \in \mathcal{C}(\overline{\mathbb{D}})$, let $f \in \mathcal{F}_{g}(\mathbb{D})$. If $f=P[f]-G[g]$ is a $K$-quasiconformal mapping with $M=\sup _{\theta \in[0,2 \pi]} \ell_{f}^{*}(\theta, 1)<+\infty$, then

$$
\left|a_{n}\right|+\left|b_{n}\right| \leq K M+\frac{2}{3}\|g\|_{\infty} \text { for } n \geq 1,
$$

where $P[f](z)=\sum_{n=0}^{\infty} a_{n} z^{n}+\sum_{n=1}^{\infty} \bar{b}_{n} \bar{z}^{n}$. In particular, if $K=1$ and $\|g\|_{\infty}=0$, then the estimate (1.13) is sharp and the extreme function is $f(z)=M z$.

The proofs of Theorems 1.1 1.5 will be presented in Section 2 .

\section{The PRoOF OF The MAIN RESUlts}

The following result easily follows from [14, Lemma 2.7].

Lemma E. If $g \in \mathcal{C}(\overline{\mathbb{D}})$, then, for $z \in \mathbb{D}$,

$$
\max \left\{\left|\frac{\partial}{\partial z} G[g](z)\right|,\left|\frac{\partial}{\partial \bar{z}} G[g](z)\right|\right\} \leq \frac{1}{3}\|g\|_{\infty},
$$

where $G[g]$ is defined in (1.4).

Proof of Theorem 1.1. We first prove the necessity. Let $z \in \Omega$ and $r=d_{\Omega}(z) / 2$. For $w \in \mathbb{D}(z, r)$, we have

$$
f(w)=J_{1}(w)-J_{2}(w)
$$

where

$$
J_{1}(w)=\frac{1}{2 \pi} \int_{0}^{2 \pi} P\left(\frac{w-z}{r}, e^{i \theta}\right) f\left(z+r e^{i \theta}\right) d \theta
$$

and

$$
J_{2}(w)=\frac{r^{2}}{2 \pi} \int_{\mathbb{D}} G\left(\frac{w-z}{r}, \zeta\right) g(r \zeta+z) d A(\zeta),
$$

where $G$ and $P$ are defined in (1.1) and (1.2), respectively. By elementary calculations, we have

$$
\frac{\partial}{\partial w} G\left(\frac{w-z}{r}, \zeta\right)=\frac{1}{2} \frac{r\left(|\zeta|^{2}-1\right)}{[r-(w-z) \bar{\zeta}](w-z-r \zeta)}
$$


and

$$
\frac{\partial}{\partial \bar{w}} G\left(\frac{w-z}{r}, \zeta\right)=\frac{1}{2} \frac{r\left(|\zeta|^{2}-1\right)}{[r-(\bar{w}-\bar{z}) \zeta](\bar{w}-\bar{z}-r \bar{\zeta})}
$$

which give that

$$
\begin{aligned}
\left\|D_{J_{2}}(w)\right\|= & \left|\frac{r^{2}}{4 \pi} \int_{\mathbb{D}} \frac{\partial}{\partial w} G\left(\frac{w-z}{r}, \zeta\right) g(r \zeta+z) d A(\zeta)\right| \\
& +\left|\frac{r^{2}}{4 \pi} \int_{\mathbb{D}} \frac{\partial}{\partial \bar{w}} G\left(\frac{w-z}{r}, \zeta\right) g(r \zeta+z) d A(\zeta)\right| \\
\leq & \frac{r^{2}\|g\|_{\infty}}{4 \pi} \int_{\mathbb{D}} \frac{1-|\zeta|^{2}}{\left|\frac{w-z}{r}-\zeta\right|\left|1-\frac{(w-z) \bar{\zeta}}{r}\right|} d A(\zeta) \\
& +\frac{r^{2}\|g\|_{\infty}}{4 \pi} \int_{\mathbb{D}} \frac{1-|\zeta|^{2}}{\left|\frac{\bar{w}-\bar{z}}{r}-\bar{\zeta}\right|\left|1-\frac{(\bar{w}-\bar{z}) \zeta}{r}\right|} d A(\zeta) .
\end{aligned}
$$

By (2.1), Lemma E and by letting $\xi=\frac{w-z}{r}$, we see that

$$
\left\|D_{J_{2}}(w)\right\| \leq \frac{r^{2}\|g\|_{\infty}}{2 \pi} \int_{\mathbb{D}} \frac{1-|\zeta|^{2}}{|\xi-\zeta||1-\xi \bar{\zeta}|} d A(\zeta) \leq \frac{2}{3}\|g\|_{\infty} r^{2} .
$$

The elementary computations lead to

$$
\frac{\partial}{\partial w} P\left(\frac{w-z}{r}, e^{i \theta}\right)=\frac{-(\bar{w}-\bar{z})\left|w-z-r e^{i \theta}\right|^{2}-\left(r^{2}-|w-z|^{2}\right)\left(\bar{w}-\bar{z}-r e^{-i \theta}\right)}{\left|w-z-r e^{i \theta}\right|^{4}},
$$

and

$$
\frac{\partial}{\partial \bar{w}} P\left(\frac{w-z}{r}, e^{i \theta}\right)=\frac{-(w-z)\left|w-z-r e^{i \theta}\right|^{2}-\left(r^{2}-|w-z|^{2}\right)\left(w-z-r e^{i \theta}\right)}{\left|w-z-r e^{i \theta}\right|^{4}} .
$$

Then, for $w \in \mathbb{D}(z, r / 2)$,

$$
\begin{aligned}
\left|\frac{\partial}{\partial w} P\left(\frac{w-z}{r}, e^{i \theta}\right)\right| & \leq \frac{|w-z|}{\left|w-z-r e^{i \theta}\right|^{2}}+\frac{r^{2}-|w-z|^{2}}{\left|w-z-r e^{i \theta}\right|^{3}} \\
& \leq \frac{\frac{r}{2}}{\frac{r^{2}}{4}}+\frac{r^{2}}{\frac{r^{3}}{8}}=\frac{10}{r}
\end{aligned}
$$

and

$$
\left|\frac{\partial}{\partial \bar{w}} P\left(\frac{w-z}{r}, e^{i \theta}\right)\right| \leq \frac{10}{r} .
$$

It follows from (2.2), (2.3) and (2.4) that, for $w \in \mathbb{D}(z, r / 2)$, 


$$
\begin{aligned}
\left\|D_{f}(w)\right\| \leq & \left|\frac{1}{2 \pi} \int_{0}^{2 \pi} \frac{\partial}{\partial w} P\left(\frac{w-z}{r}, e^{i \theta}\right)\left(f\left(z+r e^{i \theta}\right)-f(z)\right) d \theta\right| \\
& +\left|\frac{1}{2 \pi} \int_{0}^{2 \pi} \frac{\partial}{\partial \bar{w}} P\left(\frac{w-z}{r}, e^{i \theta}\right)\left(f\left(z+r e^{i \theta}\right)-f(z)\right) d \theta\right| \\
& +\left\|D_{J_{2}}(w)\right\| \\
\leq & \frac{10}{r \pi} \int_{0}^{2 \pi}\left|f\left(z+r e^{i \theta}\right)-f(z)\right| d \theta+\frac{2}{3}\|g\|_{\infty} r^{2} .
\end{aligned}
$$

Since $f \in \mathcal{L}_{\omega}(\Omega)$, we know that there is a positive constant $C_{1}$ such that

$$
\left|f\left(z+r e^{i \theta}\right)-f(z)\right| \leq C_{1} \omega(r) .
$$

Since $\Omega$ is a bounded domain, we see that there is a positive constant $C_{2}$ such that

$$
\frac{\omega(r)}{r} \geq \frac{\omega(\operatorname{diam}(\Omega))}{\operatorname{diam}(\Omega)} \geq \frac{2}{3}\|g\|_{\infty} C_{2} .
$$

By (2.5), (2.6) and (2.7), we conclude that there is a positive constant $C$ such that

$$
\left\|D_{f}(w)\right\| \leq C \frac{\omega(r)}{r} .
$$

Next, we show that the sufficiency. Since $\Omega$ is a $\mathcal{L}_{\omega}$-extension domain, we see that for any $z_{1}, z_{2} \in \Omega$, by using (1.8), there is a rectifiable curve $\gamma \subset \Omega$ joining $z_{1}$ to $z_{2}$ such that

$$
\left|f\left(z_{1}\right)-f\left(z_{2}\right)\right| \leq \int_{\gamma}\left\|D_{f}(\zeta)\right\| d s(\zeta) \leq C \int_{\gamma} \frac{\omega\left(d_{\Omega}(\zeta)\right)}{d_{\Omega}(\zeta)} d s(\zeta) \leq C \omega\left(\left|z_{1}-z_{2}\right|\right)
$$

for some constant $C>0$. The proof of this theorem is complete.

Lemma 2.1. For a given $g \in \mathcal{C}(\overline{\mathbb{D}})$, let $f \in \mathcal{F}_{g}(\mathbb{D})$. Then, for $a \in \mathbb{D}$, there is $a$ positive constant $C$ such that

$$
\left\|D_{f}(a)\right\| \leq \frac{1}{\pi r} \int_{0}^{2 \pi}\left|f\left(a+r e^{i \theta}\right)-f(a)\right| d \theta+\frac{2\|g\|_{\infty}}{3} r,
$$

where $r \in(0,1-|a|)$.

Proof. For $z \in \mathbb{D}_{r}$, let

$$
F(z)=f(z+a)-f(a) .
$$

Then, $z \in \mathbb{D}_{r}$,

$$
\Delta F(z)=\Delta f(z+a)=g(z+a) .
$$

By (1.3), we have

$$
F(z)=\frac{1}{2 \pi} \int_{0}^{2 \pi} \frac{r^{2}-|z|^{2}}{\left|z-r e^{i \theta}\right|^{2}} F\left(r e^{i \theta}\right) d \theta-\frac{r^{2}}{2 \pi} \int_{\mathbb{D}} \log \left|\frac{r-z \bar{w}}{z-r w}\right| g(r w+a) d A(w)
$$

for $z \in \mathbb{D}_{r}$. By calculations, we have 


$$
\begin{aligned}
F_{z}(z)= & \frac{1}{2 \pi} \int_{0}^{2 \pi} \frac{-\bar{z}\left|z-r e^{i \theta}\right|^{2}-\left(r^{2}-|z|^{2}\right)\left(\bar{z}-r e^{-i \theta}\right)}{\left|z-r e^{i \theta}\right|^{4}} F\left(r e^{i \theta}\right) d \theta \\
& -\frac{r^{3}}{4 \pi} \int_{\mathbb{D}} \frac{\left(|w|^{2}-1\right)}{(r-z \bar{w})(z-r w)} g(r w+a) d A(w)
\end{aligned}
$$

and

$$
\begin{aligned}
F_{\bar{z}}(z)= & \frac{1}{2 \pi} \int_{0}^{2 \pi} \frac{-z\left|z-r e^{i \theta}\right|^{2}-\left(r^{2}-|z|^{2}\right)\left(z-r e^{i \theta}\right)}{\left|z-r e^{i \theta}\right|^{4}} F\left(r e^{i \theta}\right) d \theta \\
& -\frac{r^{3}}{4 \pi} \int_{\mathbb{D}} \frac{\left(|w|^{2}-1\right)}{(r-w \bar{z})(\bar{z}-r \bar{w})} g(r w+a) d A(w),
\end{aligned}
$$

which yields that

$$
\begin{aligned}
\left\|D_{F}(0)\right\| & \leq \frac{1}{r \pi} \int_{0}^{2 \pi}\left|F\left(r e^{i \theta}\right)\right| d \theta+\frac{r}{2 \pi} \int_{\mathbb{D}} \frac{\left(1-|w|^{2}\right)}{|w|}|g(r w+a)| d A(w) \\
& \leq \frac{1}{r \pi} \int_{0}^{2 \pi}\left|F\left(r e^{i \theta}\right)\right| d \theta+\frac{2\|g\|_{\infty}}{3} r .
\end{aligned}
$$

The proof of this lemma is complete.

Proof of Theorem 1.2. We first prove $(1) \Rightarrow(2)$. By Lemma 2.1, for $\rho \in(0, d(z)]$,

$$
\left\|D_{f}(z)\right\| \leq \frac{1}{\pi \rho} \int_{0}^{2 \pi}\left|f\left(z+\rho e^{i \theta}\right)-f(z)\right| d \theta+\frac{2\|g\|_{\infty}}{3} \rho,
$$

which gives

$$
\begin{aligned}
\int_{0}^{r} \rho^{2}\left\|D_{f}(z)\right\| d \rho \leq & \frac{1}{\pi} \int_{0}^{r}\left(\rho \int_{0}^{2 \pi}\left|f\left(z+\rho e^{i \theta}\right)-f(z)\right| d \theta\right) d \rho \\
& +\frac{2\|g\|_{\infty}}{3} \int_{0}^{r} \rho^{3} d \rho
\end{aligned}
$$

where $r=d(z)$. It follows from (2.8) that

$$
\begin{aligned}
\left\|D_{f}(z)\right\| & \leq \frac{3}{\pi r^{3}} \int_{\mathbb{D}(z, r)}|f(z)-f(\zeta)| d A(\zeta)+\frac{\|g\|_{\infty}}{2} r \\
& =\frac{3}{r|\mathbb{D}(z, r)|} \int_{\mathbb{D}(z, r)}|f(z)-f(\zeta)| d A(\zeta)+\frac{\|g\|_{\infty}}{2} r \\
& \leq \frac{3 C}{\omega\left(r^{\alpha}\right)}+\frac{\|g\|_{\infty}}{2} r,
\end{aligned}
$$

which gives that $f \in \mathcal{B}_{\omega}^{\alpha}$. 
Now we prove $(2) \Rightarrow(1)$. Since $f \in \mathcal{B}_{\omega}^{\alpha}$, we see that there is a positive constant $C$ such that

$$
\left\|D_{f}(z)\right\| \leq \frac{C}{\omega\left(d^{\alpha}(z)\right)}
$$

For $z \in \mathbb{D}$ and $\zeta \in \mathbb{D}(z, r)$, we have

$$
\omega\left(d^{\alpha}(z+t(\zeta-z))\right) \geq \omega\left((d(z)-t|z-\zeta|)^{\alpha}\right), t \in[0,1],
$$

which, together with (2.9), yields that

$$
\begin{aligned}
|f(z)-f(\zeta)| & \leq|z-\zeta| \int_{0}^{1}\left\|D_{f}(z+t(\zeta-z))\right\| d t \\
& \leq C|z-\zeta| \int_{0}^{1} \frac{d t}{\omega\left(d^{\alpha}(z+t(\zeta-z))\right)} \\
& \leq C|z-\zeta| \int_{0}^{1} \frac{d t}{\omega\left((d(z)-t|z-\zeta|)^{\alpha}\right)} \\
& =C \int_{0}^{|z-\zeta|} \frac{d t}{\omega\left((d(z)-t)^{\alpha}\right)} .
\end{aligned}
$$

By (2.10), we conclude that

$$
\begin{aligned}
\frac{1}{|\mathbb{D}(z, r)|} \int_{\mathbb{D}(z, r)}|f(z)-f(\zeta)| d A(\zeta) & \leq \frac{C}{\left|\mathbb{D}_{r}\right|} \int_{\mathbb{D}_{r}}\left(\int_{0}^{|\xi|} \frac{d t}{\omega\left((d(z)-t)^{\alpha}\right)}\right) d A(\xi) \\
& =\frac{2 C}{r^{2}} \int_{0}^{r} \rho\left(\int_{0}^{\rho} \frac{d t}{\omega\left((d(z)-t)^{\alpha}\right)}\right) d \rho .
\end{aligned}
$$

By exchanging integral order, we obtain

$$
\begin{aligned}
\int_{0}^{r} \rho\left(\int_{0}^{\rho} \frac{d t}{\omega\left((d(z)-t)^{\alpha}\right)}\right) d \rho & =\int_{0}^{r}\left(\int_{t}^{r} \rho d \rho\right) \frac{d t}{\omega\left((r-t)^{\alpha}\right)} \\
& \leq r \int_{0}^{r} \frac{(r-t)^{\alpha}}{\omega\left((r-t)^{\alpha}\right)}(r-t)^{1-\alpha} d t \\
& \leq \frac{r^{\alpha+1}}{\omega\left(r^{\alpha}\right)} \int_{0}^{r}(r-t)^{1-\alpha} d t \\
& =\frac{1}{2-\alpha} \frac{r^{3}}{\omega\left(r^{\alpha}\right)}
\end{aligned}
$$

It follows from (2.11) and (2.12) that

$$
\frac{1}{|\mathbb{D}(z, r)|} \int_{\mathbb{D}(z, r)}|f(z)-f(\zeta)| d A(\zeta) \leq \frac{2 C}{2-\alpha} \frac{r}{\omega\left(r^{\alpha}\right)} .
$$

The proof of this theorem is complete.

The following result is well-known (cf. [3]). 
Lemma F. Among all rectifiable Jordan curves of a given length, the circle has the maximum interior area.

Proof of Theorem 1.4. We first prove (1.10). Since $P[f]$ is harmonic in $\mathbb{D}$, we see that $\partial P[f](z) / \partial z$ and $\partial P[f](z) / \partial \bar{z}$ are analytic and anti-analytic, respectively. Hence, by Cauchy's integral formula, we have

$$
n a_{n}=\frac{1}{2 \pi i} \int_{|z|=r} \frac{\frac{\partial P[f](z)}{\partial z}}{z^{n}} d z \text { and } n b_{n}=\frac{1}{2 \pi i} \int_{|z|=r} \frac{\overline{\left(\frac{\partial P[f](z)}{\partial \bar{z}}\right)}}{z^{n}} d z,
$$

which, together with $\left\|D_{P[f]}\right\| \leq\left\|D_{f}\right\|+\left\|D_{G[g]}\right\|$, implies that

$$
\begin{aligned}
n\left(\left|a_{n}\right|+\left|b_{n}\right|\right) & =\frac{1}{2 \pi}\left(\left|\int_{|z|=r} \frac{\frac{\partial P[f](z)}{\partial z}}{z^{n}} d z\right|+\left|\int_{|z|=r} \frac{\overline{\left(\frac{\partial P[f](z)}{\partial \bar{z}}\right)}}{z^{n}} d z\right|\right) \\
& \leq \frac{1}{2 \pi r^{n}} \int_{0}^{2 \pi} r\left\|D_{P[f]}\left(r e^{i \theta}\right)\right\| d \theta \\
& \leq \frac{1}{2 \pi r^{n}} \int_{0}^{2 \pi} r\left(\left\|D_{f}\left(r e^{i \theta}\right)\right\|+\left\|D_{G[g]}\left(r e^{i \theta}\right)\right\|\right) d \theta
\end{aligned}
$$

where $r \in(0,1)$.

By (1.9), we have

$$
\begin{aligned}
\ell_{f}(1) & \geq \ell_{f}(r)=r \int_{0}^{2 \pi}\left|f_{z}\left(r e^{i \theta}\right)-e^{-2 i \theta} f_{\bar{z}}\left(r e^{i \theta}\right)\right| d \theta \\
& \geq r \int_{0}^{2 \pi}\left(\left|f_{z}\left(r e^{i \theta}\right)\right|-\left|f_{\bar{z}}\left(r e^{i \theta}\right)\right|\right) d \theta \\
& \geq \frac{r}{K} \int_{0}^{2 \pi}\left\|D_{f}\left(r e^{i \theta}\right)\right\| d \theta .
\end{aligned}
$$

It follows from (2.13), (2.14) and Lemma E that

$$
\begin{aligned}
n\left(\left|a_{n}\right|+\left|b_{n}\right|\right) & \leq \frac{K \ell_{f}(1)}{2 \pi r^{n}}+\frac{1}{2 \pi r^{n}} \int_{0}^{2 \pi} r\left\|D_{G[g]}\left(r e^{i \theta}\right)\right\| d \theta \\
& \leq \frac{1}{2 \pi r^{n}}\left(K \ell_{f}(1)+\int_{0}^{2 \pi}\left\|D_{G[g]}\left(r e^{i \theta}\right)\right\| d \theta\right) \\
& \leq \frac{1}{2 \pi r^{n}}\left(K \ell_{f}(1)+\frac{4 \pi}{3}\|g\|_{\infty}\right),
\end{aligned}
$$

which gives that

$$
\left|a_{n}\right|+\left|b_{n}\right| \leq \inf _{r \in(0,1)}\left[\frac{1}{2 n \pi r^{n}}\left(K \ell_{f}(1)+\frac{4 \pi}{3}\|g\|_{\infty}\right)\right]=\frac{K \ell_{f}(1)}{2 n \pi}+\frac{2}{3 n}\|g\|_{\infty} .
$$


Next we prove (1.11). Let Area $\left(f\left(\mathbb{D}_{r}\right)\right)$ denote the area of $f\left(\mathbb{D}_{r}\right)$, where $r \in(0,1)$. Then

$$
\operatorname{Area}\left(f\left(\mathbb{D}_{r}\right)\right)=\int_{\mathbb{D}_{r}} J_{f}(z) d A(z) \geq \frac{1}{K} \int_{\mathbb{D}_{r}}\left\|D_{f}(z)\right\|^{2} d A(z) .
$$

For $\theta \in[0,2 \pi]$ and $z \in \mathbb{D}$, let

$$
H_{\theta}(z)=\frac{\partial P[f](z)}{\partial z}+e^{i \theta} \overline{\left(\frac{\partial P[f](z)}{\partial \bar{z}}\right)} .
$$

Then, by the subharmonicity of $\left|H_{\theta}\right|^{2}$, we obtain

$$
\begin{aligned}
\left|H_{\theta}(z)\right|^{2} & \leq \frac{1}{\pi\left(1-|z|^{2}\right)^{2}} \int_{0}^{1-|z|^{2}} \rho \int_{0}^{2 \pi}\left|H_{\theta}\left(z+\rho e^{i \gamma}\right)\right|^{2} d \gamma d \rho \\
& \leq \frac{1}{\pi\left(1-|z|^{2}\right)^{2}} \int_{\mathbb{D}_{1-|z|^{2}}}\left\|D_{P[f]}(z+\zeta)\right\|^{2} d A(\zeta) \\
& \leq \frac{I}{\pi\left(1-|z|^{2}\right)^{2}}
\end{aligned}
$$

where

$$
I=\int_{\mathbb{D}}\left(\left\|D_{G[g]}(\xi)\right\|+\left\|D_{f}(\xi)\right\|\right)^{2} d A(\xi) .
$$

By (2.15), Lemma E and Cauchy-Schwarz's inequality, we get

$$
\begin{aligned}
I= & \int_{\mathbb{D}}\left\|D_{f}(\xi)\right\|^{2} d A(\xi)+\int_{\mathbb{D}}\left\|D_{G[g]}(\xi)\right\|^{2} d A(\xi) \\
& +2 \int_{\mathbb{D}}\left\|D_{f}(\xi)\right\|\left\|D_{G[g]}(\xi)\right\| d A(\xi) \\
\leq & \int_{\mathbb{D}}\left\|D_{f}(\xi)\right\|^{2} d A(\xi)+\frac{4 \pi}{9}\|g\|_{\infty}^{2}+\frac{2}{3}\|g\|_{\infty} \int_{\mathbb{D}}\left\|D_{f}(\xi)\right\| d A(\xi) \\
\leq & K \operatorname{Area}(f(\mathbb{D}))+\frac{4 \pi}{9}\|g\|_{\infty}^{2} \\
& +\frac{2}{3}\|g\|_{\infty}\left(\int_{\mathbb{D}}\left\|D_{f}(\xi)\right\|^{2} d A(\xi)\right)^{\frac{1}{2}}\left(\int_{\mathbb{D}} d A(\xi)\right)^{\frac{1}{2}} \\
\leq & K \operatorname{Area}(f(\mathbb{D}))+\frac{4 \pi}{9}\|g\|_{\infty}^{2}+\frac{2 \pi^{\frac{1}{2}}}{3}\|g\|_{\infty}(K \operatorname{Area}(f(\mathbb{D})))^{\frac{1}{2}} .
\end{aligned}
$$

Applying Lemma F, we have

$$
\operatorname{Area}(f(\mathbb{D})) \leq \pi\left(\frac{\ell_{f}(1)}{2 \pi}\right)^{2}=\frac{\ell_{f}^{2}(1)}{4 \pi},
$$

which, together with (2.17), yields that 


$$
I \leq \frac{\ell_{f}^{2}(1) K}{4 \pi}+\frac{4 \pi}{9}\|g\|_{\infty}^{2}+\frac{\ell_{f}(1) K^{\frac{1}{2}}}{3}\|g\|_{\infty} .
$$

By (2.16) and (2.18), we conclude that

$$
\left\|D_{P[f]}(z)\right\|=\max _{\theta \in[0,2 \pi]}\left|H_{\theta}(z)\right| \leq \frac{\left(\frac{\ell_{f}^{2}(1) K}{4 \pi^{2}}+\frac{4}{9}\|g\|_{\infty}^{2}+\frac{\ell_{f}(1) K^{\frac{1}{2}}}{3 \pi}\|g\|_{\infty}\right)^{\frac{1}{2}}}{1-|z|^{2}} .
$$

At last, $f \in \mathcal{B}_{\omega}^{1}$ follows from (2.19) and Lemma E, where $\omega(t)=t$. The proof of this theorem is complete.

The following result is considered to be a Schwarz-type lemma of subharmonic functions.

Theorem G. ([2, Theorem 2]) Let $\phi$ be subharmonic in $\mathbb{D}$. If, for all $r \in[0,1)$,

$$
A(r)=\sup _{\theta \in[0,2 \pi]} \int_{0}^{r} \phi\left(\rho e^{i \theta}\right) d \rho \leq 1
$$

then $A(r) \leq r$.

Proof of Theorem 1.5. By Cauchy's integral formula, for $\rho \in(0,1)$ and $n \geq 1$, we get

$$
n a_{n}=\frac{1}{2 \pi i} \int_{|z|=\rho} \frac{\frac{\partial P[f](z)}{\partial z}}{z^{n}} d z \text { and } n b_{n}=\frac{1}{2 \pi i} \int_{|z|=\rho} \frac{\overline{\left(\frac{\partial P[f](z)}{\partial z}\right)}}{z^{n}} d z,
$$

which implies that

$$
\begin{aligned}
n\left(\left|a_{n}\right|+\left|b_{n}\right|\right) & =\frac{1}{2 \pi}\left|\int_{|z|=\rho} \frac{\frac{\partial P[f](z)}{\partial z}}{z^{n}} d z\right|+\frac{1}{2 \pi}\left|\int_{|z|=\rho} \frac{\overline{\left(\frac{\partial P[f](z)}{\partial \bar{z}}\right)}}{z^{n}} d z\right| \\
& \leq \frac{1}{2 \pi \rho^{n-1}} \int_{0}^{2 \pi}\left\|D_{P[f]}\left(\rho e^{i \theta}\right)\right\| d \theta
\end{aligned}
$$

By calculations, for $\theta \in[0,2 \pi]$, we obtain

$$
\begin{aligned}
\ell_{f}^{*}(\theta, r) & =\int_{0}^{r}\left|f_{z}\left(\rho e^{i \theta}\right)+e^{-2 i \theta} f_{\bar{z}}\left(\rho e^{i \theta}\right)\right| d \rho \\
& \geq \int_{0}^{r} \lambda\left(D_{f}\right)\left(\rho e^{i \theta}\right) d \rho \\
& \geq \frac{1}{K} \int_{0}^{r}\left\|D_{f}\left(\rho e^{i \theta}\right)\right\| d \rho,
\end{aligned}
$$

which gives

$$
\int_{0}^{r}\left\|D_{f}\left(\rho e^{i \theta}\right)\right\| d \rho \leq K \ell_{f}^{*}(\theta, r) \leq K M .
$$


It follows from (2.21) and Lemma E that

$$
\begin{aligned}
\int_{0}^{r}\left\|D_{P[f]}\left(\rho e^{i \theta}\right)\right\| d \rho & \leq \int_{0}^{r}\left\|D_{f}\left(\rho e^{i \theta}\right)\right\| d \rho+\int_{0}^{r}\left\|D_{G[g]}\left(\rho e^{i \theta}\right)\right\| d \rho \\
& \leq K M+\frac{2}{3}\|g\|_{\infty} r .
\end{aligned}
$$

By (2.21), the subharmonicity of $D_{P[f]}\left(\rho e^{i \theta}\right)$ and Theorem $\mathrm{G}$, we have

$$
\int_{0}^{r}\left\|D_{P[f]}\left(\rho e^{i \theta}\right)\right\| d \rho \leq\left(K M+\frac{2}{3}\|g\|_{\infty}\right) r
$$

By (2.20) and (2.23), we get

$$
\begin{aligned}
2 \pi n\left(\left|a_{n}\right|+\left|b_{n}\right|\right) \int_{0}^{r} \rho^{n-1} d \rho & =\int_{0}^{r}\left(\int_{0}^{2 \pi}\left\|D_{P[f]}\left(\rho e^{i \theta}\right)\right\| d \theta\right) d \rho \\
& =\int_{0}^{2 \pi}\left(\int_{0}^{r}\left\|D_{P[f]}\left(\rho e^{i \theta}\right)\right\| d \theta\right) d \rho \\
& \leq 2 \pi\left(K M+\frac{2}{3}\|g\|_{\infty}\right) r
\end{aligned}
$$

which yields that

$$
\left|a_{n}\right|+\left|b_{n}\right| \leq \inf _{r \in(0,1)}\left(\frac{K M+\frac{2}{3}\|g\|_{\infty}}{r^{n-1}}\right)=K M+\frac{2}{3}\|g\|_{\infty} \text { for } n \geq 1 .
$$

The proof of this theorem is complete.

Acknowledgements: This research was partly supported by the Science and Technology Plan Project of Hengyang City (No. 2018KJ125), the National Natural Science Foundation of China (No. 11571216), the Science and Technology Plan Project of Hunan Province (No. 2016TP1020), the Science and Technology Plan Project of Hengyang City (No. 2017KJ183), and the Application-Oriented Characterized Disciplines, Double First-Class University Project of Hunan Province (Xiangjiaotong [2018]469).

\section{REFERENCES}

1. A. Abaob, M. Arsenovic and M. Mateljević, Moduli of continuity of harmonic quasiregular mappings on bounded domains, Ann. Acad. Sci. Fenn. Math., 38 (2013), 839-847.

2. E. F. Beckenbach, A relative of the lemma of Schwarz, Bull. Amer. Math. Soc., 44 (1938), 698-707.

3. T. Carleman, Zur Theorie der Minimalflächen, Math. Z. 9(1921), 154-160.

4. Sh. Chen, G. Liu and S. Ponnusamy, Linear measure and K-quasiconformal harmonic mappings (in Chinese), Sci. Sin. Math., 47(2017), 565-574.

5. Sh. Chen, S. Ponnusamy and A. Rasila, On characterizations of Bloch-type, Hardy-type and Lipschitz-type spaces, Math. Z., 279(2015), 163-183.

6. Sh. Chen, S. Ponnusamy and A. Rasila, Lengths, area and Lipschitz-type spaces of planar harmonic mappings, Nonlinear Anal., 115(2015), 62-80.

7. P. Duren, Theory of $H^{p}$ spaces, 2nd ed., Dover, Mineola, N. Y., 2000. 
8. K. M. Dyakonov, Holomorphic functions and quasiconformal mappings with smooth moduli, Adv. Math., 187 (2004), 146-172.

9. K. M. Dyakonov, Equivalent norms on Lipschitz-type spaces of holomorphic functions, Acta Math., 178 (1997), 143-167.

10. F. W. Gehring and O. Martio, Lipschitz-classes and quasiconformal mappings, Ann. Acad. Sci. Fenn. Ser. A I Math., 10 (1985), 203-219.

11. F. Holland and D. Walsh, Criteria for membership of Bloch space and its subspace, BMOA, Math. Ann., 273(1986), 317-335.

12. L. Hörmander, Notions of convexity, Progress in Mathematics, Vol. 127, Birkhäuser Boston Inc, Boston 1994.

13. D. Kalaj and M. Pavlović, On quasiconformal self-mappings of the unit disk satisfying Poisson's equation, Trans. Amer. Math. Soc., 363 (2011), 4043-4061.

14. D. Kalaj, Cauchy transform and Poisson's equation, Adv. Math., 231 (2012), 213-242.

15. S. G. Krantz, Lipschitz spaces, smoothness of functions, and approximation theory, Expo. Math., 3 (1983), 193-260.

16. V. Lappalainen, Lip ${ }_{h}$-extension domains, Ann. Acad. Sci. Fenn. Ser. A I Math. Dissertationes, 56 (1985).

17. M. Mateljević and M. Vuorinen, On harmonic quasiconformal quasi-isometries, J. Inequa. Appl., Volume 2010, Article ID 178732, 19 pages, doi:10.1155/2010/178732.

18. M. Mateljević, Distortion of quasiregular mappings and equivalent norms on Lipschitztype spaces, Abstr. Appl. Anal., Volume 2014, Article ID 895074, 20 pages, http://dx.doi.org/10.1155/2014/895074.

19. M. Pavlović, On the Holland-Walsh characterization of Bloch functions, Proc. Edinb. Math. Soc., 51 (2008), 439-441.

20. M. Pavlović, On Dyakonov's paper Equivalent norms on Lipschitz-type spaces of holomorphic functions, Acta Math., 183(1999), 141-143.

21. G. Ren and U. Kähler, Weighted Lipschitz continuity and harmonic Bloch and Besov spaces in the real unit ball, Proc. Edinb. Math. Soc., 48 (2005), 743-755.

Sh. Chen, College of Mathematics and Statistics, Hengyang Normal University, Hengyang, Hunan 421008, People's Republic of China.

E-mail address: mathechen@126.com 